\title{
Decorin: a multifunctional proteoglycan involved in oocyte maturation and trophoblast migration
}

\author{
Beom Seok Park ${ }^{1,2,3}$, Jaewang Lee ${ }^{1,2}$, Jin Hyun Jun ${ }^{1,2,3,4}$ \\ ${ }^{1}$ Department of Biomedical Laboratory Science, College of Health Science, Eulji University, Seongnam; ${ }^{2}$ Department of Biomedical Laboratory Science, \\ Graduate School, Eulji University, Seongnam; ${ }^{3}$ Department of Senior Healthcare, BK21 Plus Program, Graduate School, Eulji University, Seongnam; ${ }^{4}$ Eulji \\ Medi-Bio Research Institute (EMBRI), Eulji University, Daejeon, Korea
}

Decorin (DCN) is a proteoglycan belonging to the small leucine-rich proteoglycan family. It is composed of a protein core containing leucine repeats with a glycosaminoglycan chain consisting of either chondroitin sulfate or dermatan sulfate. DCN is a structural component of connective tissues that can bind to type I collagen. It plays a role in the assembly of the extracellular matrix (ECM), and it is related to fibrillogenesis. It can interact with fibronectin, thrombospondin, complement component C1, transforming growth factor (TGF), and epidermal growth factor receptor. Normal DCN expression regulates a wide range of cellular processes, including proliferation, migration, apoptosis, and autophagy, through interactions with various molecules. However, its aberrant expression is associated with oocyte maturation, oocyte quality, and poor extravillous trophoblast invasion of the uterus, which underlies the occurrence of preeclampsia and intrauterine growth restriction. Spatiotemporal hormonal control of successful pregnancy should regulate the concentration and activity of specific proteins such as proteoglycan participating in the ECM remodeling of trophoblastic and uterine cells in fetal membranes and uterus. At the human feto-maternal interface, TGF- $\beta$ and DCN play crucial roles in the regulation of trophoblast invasion of the uterus. This review summarizes the role of the proteoglycan DCN as an important and multifunctional molecule in the physiological regulation of oocyte maturation and trophoblast migration. This review also shows that recombinant $D C N$ proteins might be useful for substantiating diverse functions in both animal and in vitro models of oogenesis and implantation.

Keywords: Decorin; Extracellular matrix; Implantation; Oocyte; Oogenesis; Proteoglycan; Trophoblasts

\section{Characteristics and functions of decorin}

About 20 years ago, proteoglycan gene families were classified and simplified according to three criteria: their cellular and subcellular location, the overall gene and protein homology, and the presence of specific protein modules within their respective protein

Received: November 4, 2021 · Revised: November 11, 2021 · Accepted: November 15, 2021 Corresponding author: Jin Hyun Jun

Department of Biomedical Laboratory Science, College of Health Science, Eulji University, 553 Sanseong-daero, Sujeong-gu, Seongnam 13135, Korea Tel: +82-31-740-7210 Fax:+82-31-740-7351 E-mail:junjh55@hanmail.net

\footnotetext{
* This study was supported by the project entitled "Exploring evolution of the structure and immune proteins of crustaceans in hydrothermal vents" funded by the Korean Ministry of Oceans and Fisheries (No. 1348201458). And, this paper was supported by Eulji University in 2021 (Beom Seok Park).

This is an Open Access article distributed under the terms of the Creative Commons Attribution Non-Commercial License (http://creativecommons.org/licenses/by-nc/4.0/) which permits unrestricted non-commercial use, distribution, and reproduction in any medium, provided the original work is properly cited.
}

cores. In particular, the largest class is formed by a set of extracellular proteoglycans encoded by 25 genes. The first group contains the 4 genes for hyalectan, a major structural component of cartilage, blood vessels, and the central nervous system. The second comprises 18 small leucine-rich proteoglycans (SLRPs). These SLRPs have a multitude of functions, including signaling through various receptors. Many SLRPs are found in the circulation and various body fluids. The third is the SPOCK family of calcium-binding heparan sulfate proteoglycans [1].

The SLRPs expressed in most extracellular matrices (ECMs) generally can act as structural components to maintain tissue architecture. They are involved in range of fundamental biological and physiological functions, including cell adhesion, signal transduction, and the immune response. SLRPs share many biological functions by binding to ECM components, particularly various collagens, receptor tyrosine kinases, and innate immune receptors (Toll-like receptors) on cell surfaces when present in a soluble form $[2,3]$. They are characterized 
by a relatively small protein core of $36-42 \mathrm{kDa}$, including leucine-rich repeats (LRRs) [4]. Individual LRR modules have 20-30 amino acid residues with a highly conserved " $L x x L x L x x N$ " motif. The central $L x L$ part of the module forms the core $\beta$ strand, with two leucines pointing towards the interior of the protein, making up the hydrophobic core, whereas the variable $x$ residues within the motif are exposed to solvent. Some are involved in interactions with ligands. Aspargines in the motif make continuous hydrogen bonds with backbone carbonyls of neighboring $\beta$-strands throughout the entire protein. This extended hydrogen bond network is called the "asparagine ladder." Therefore, $\beta$-strands are more closely packed and assembled into a large $\beta$-sheet, making up the entire concave surface of the horseshoe. Variable amino acids, except the conserved $\beta$-strands of each LRR module, are surface-exposed. Some of them play important roles in ligand interactions. To prevent the exposure of hydrophobic core of LRR modules, it has two special modules named LRRNT and LRRCT in the $\mathrm{N}$ - and C-termini of proteins. These modules do not fol- low the sequence conservation pattern of LRR modules. They often contain an anti-parallel $\beta$-hairpin stabilized by disulfide bridges [5-7].

The 18 SLRP members are grouped into five classes. Classes I-III have the conserved C-terminal cysteine-rich capping motif, a unique feature that has recently been described as the "ear repeat," appearing as an abnormal pattern of cysteine residues followed by the asparagine residue in consensus sequences. Classes IV and V are noncanonical fragments without the ear repeat $[1,2,4]$. The sequence alignment and phylogenetic tree of the SLRPs are presented in Figure 1.

Decorin (DCN), which belongs to class I, is produced by a variety of stromal cells in the body, such as fibroblasts in the dermis, cornea, and chondrocytes of cartilage. It may participate in ECM remodeling during the attachment and detachment of the placenta within the course of pregnancy in cows [8]. Those with disrupted DCN genes are viable, but they show fragile skin with markedly reduced tensile strength. As a result, aberrant collagen morphology appears in the
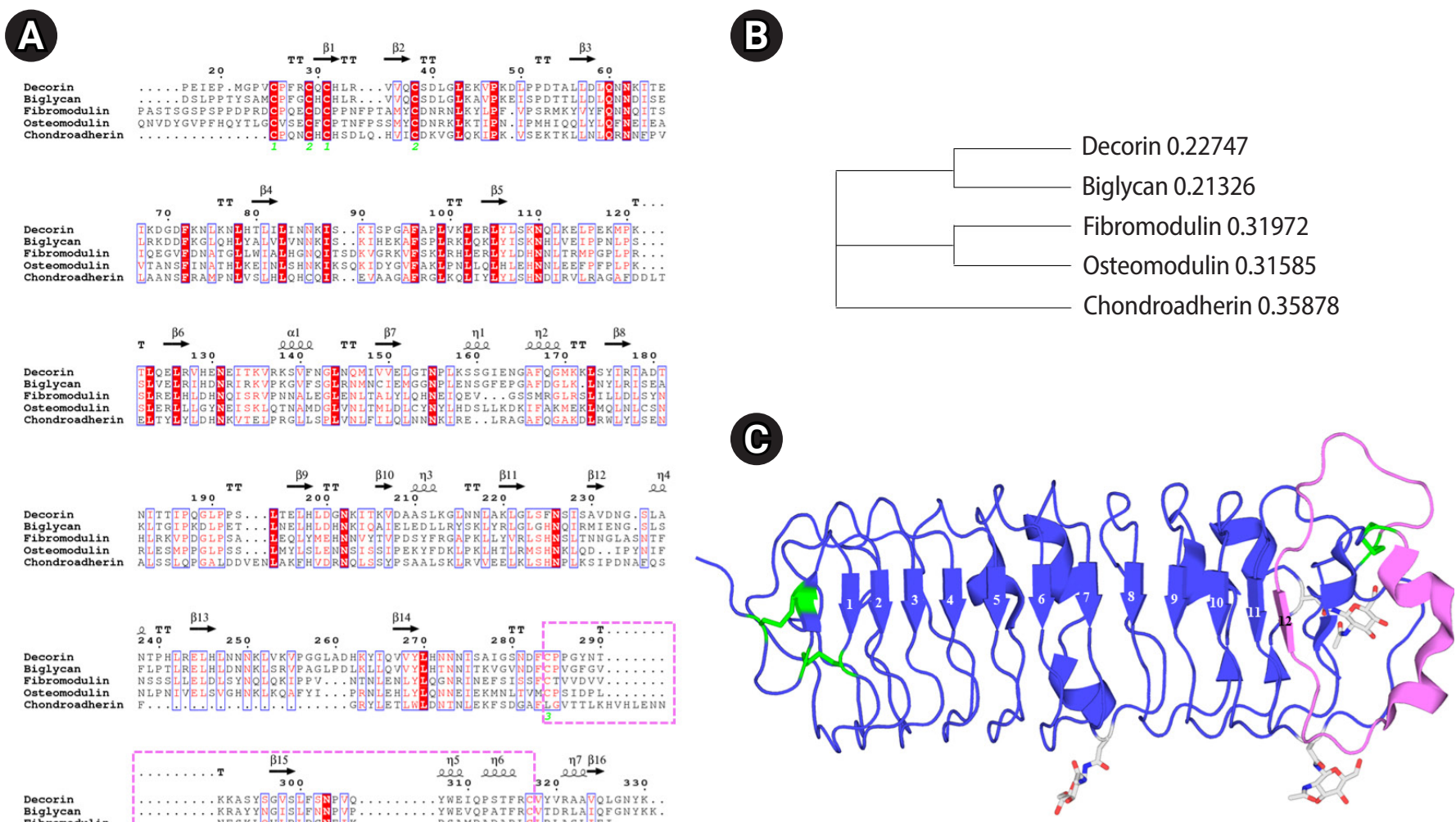

Figure 1. Sequence alignment and phylogenetic tree of small leucine-rich proteoglycans (SLRPs) using known crystal structures of decorin (PDB ID: 1XKU), biglycan (PDB ID: 2FT3), fibromodulin (PDB ID: 5MXO), osteomodulin (PDB ID: 5YQ5), and chondroadherin (PDB ID: 5LFN). (A) Sequence alignment of crystal structures of five SLRPs. Protein sequences were aligned with Clustal Omega (Clustal Omega <Multiple Sequence Alignment<EMBL-EBI) and generated using the EsPript 3.0 program (ESPript 3 [ibcp.fr]). (B) Phylogenetic tree of SLRPs with known crystal structures. (C) Diagram of the crystal structure of Bos taurus decorin rendered with PyMOL v1.8. Vertical arrows indicate $\beta$-strands. Coiled ribbons indicate $\alpha$-helices. Leucine-rich repeats (LRRs) are numbered above the $\beta$-strands. N-acetylglucosamine is linked to N182, N233, and N274. Disulfide bridges are shown in green. The terminal LRR Cys capping motif, known as the ear repeat, is highlighted in pink. 
skin and tendon with coarser and irregular fiber outlines [9]. Normal DCN expression regulates a wide range of cellular processes including proliferation, migration, apoptosis, and autophagy through interactions with various molecules. However, aberrant expression of DCN has been associated with poor extravillous trophoblast (EVT) invasion of the uterus, which underlies the occurrence of preeclampsia (PE) and intrauterine growth restriction (IUGR) [10].

During pregnancy, placental cells are under tight hormonal control. Among others, they regulate the concentration and activity of specific proteins participating in the ECM remodeling of fetal membranes [11]. Therefore, the proteoglycan DCN plays a variety of roles. The most important role of DCN is that it can regulate cell adhesion mediated by various binding proteins and tissues for fetal formation.

\section{Production of recombinant DCN protein in the laboratory}

For functional analysis, DCN should be made by recombinant DNA technology in the laboratory. The whole gene of the human DCN protein was amplified by PCR and cloned into a modified pAcGP67a vector. The cloned DNA of the DCN gene was confirmed by sequencing. Large-scale DNA preparations were then performed to obtain a sufficient amount of transfection-grade plasmid DNA, which was co-transfected with ProGreen (a baculovirus genomic vector) into Sf9 (Spodoptera frugiperda) insect cells. The vector was then generated and amplified by recombination between the cloned vector and viral DNA. The Fc tagged DCN protein was expressed in High Five cells. The culture media of the High Five cells were harvested and supernatants containing secreted DCN-Fc proteins were purified by protein A affinity chromatography. The molecular mass of the DCN protein was calculated to be approximately $37.9 \mathrm{kDa}$, with an isoelectric point ( $\mathrm{pl}$ ) of 8.61, using the theoretical tool "Compute $\mathrm{pl} / \mathrm{Mw}^{\text {" }}$ (https://web.expasy.org/compute_pi/). The DCN-Fc tag was cleaved by thrombin. Using Superdex 200-size exclusion chromatography (SEC), the cleaved DCN was further purified. It was found to be a monomer, which was validated as a SEC standard component. The purification steps of affinity and SEC were monitored by sodium dodecyl sulfate-polyacrylamide gel electrophoresis (SDS-PAGE). The above procedure in insect cells allowed the efficient generation of recombinant DCN protein, which was subsequently expressed and purified as shown in Figure 2.

\section{DCN in the ovary and oocyte maturation}

The ovary in adults has diverse functions, including follicle growth and maturation, ovulation, regression, and corpus luteum $(\mathrm{CL})$ formation. During this process, bidirectional communication between follicles and the ECM may influence the quality of oocytes in mammals. Many studies have demonstrated that DCN is present in the ECM of normal and tumorous ovaries in humans $[12,13]$. As discussed above, DCN is a secreted proteoglycan that plays a structural role in the ECM. It can interfere with the signaling of multiple growth factors and their receptors [14]. For instance, DCN can directly bind to epidermal growth factor receptor (EGFR), which has many physiological functions in oocyte maturation $[15,16]$. When DCN binds to EGFR, it causes phosphorylation and activation of EGFR, followed by internalization and downregulation of EGFR signaling, resulting in a chronic blockage of the EGF/EGFR signaling pathway [17-21]. In the ovary, EGF-like molecules, including amphiregulin and epiregulin, with roles in the orchestration of ovulation and subsequent development of the $\mathrm{CL}$ in response to the luteinizing hormone surge are produced in a fine-tuned manner [15,22]. Regarding human oocyte maturation, EGF also targets human oocytes to regulate their meiotic maturation [23]. According to a previous report, DCN is present in human and monkey ovarian stroma, follicular theca cells, luteal cells, and the follicular fluid of ovulatory follicles. It has been postulated that DCN can act as a paracrine signaling factor by inhibiting growth factor/growth factor receptor interactions, suggesting that DCN might regulate folliculogenesis and oocyte quality [24]. Peng et al. [25] synthesized full-length cDNA of goat DCN and identified its expression patterns in various tissues and the ovary. They also confirmed that over-expressed DCN not only could promote programmed cell death through a non-mitochondrial apoptosis pathway, but also could enhance cell arrest by p21 upregulation. Notably, the physiological actions of DCN are mediated through multiple signaling pathways, including the PKA, p38 kinase, and PI3K pathways. Kedem et al. [26] recently conducted a prospective study involving 49 patients treated at a local assisted reproductive technology (ART) center. They characterized the in vivo expression of DCN mRNA in mural and cumulus granulosa cells (CGCs). In previous studies, the human chorionic gonadotropin-induced expression of DCN was found to be highly upregulated in pre-ovulatory follicles [27-29]. Interestingly, DCN expression in human CGCs seems to be correlated with maturation stage of the corresponding oocyte. A plausible explanation is that oocytes that arrest at immature stages under controlled ovarian hyperstimulation finally fail to undergo maturation. Their adjacent cumulus cells also fail to express DCN. Another explanation is that downregulated DCN expression in CGCs is one of causative factors for meiotic arrest. Miscommunication between the oocyte and surrounding somatic cells may result in subfertility [26].

Sawada et al. [30] suggested that DCN in the follicular fluid (F-DCN) is a useful biomarker of the quality of oocytes retrieved from the corresponding follicles in ART. They analyzed 130 oocytes of 88 patients treated with ART because of unexplained infertility. In patients with 
A
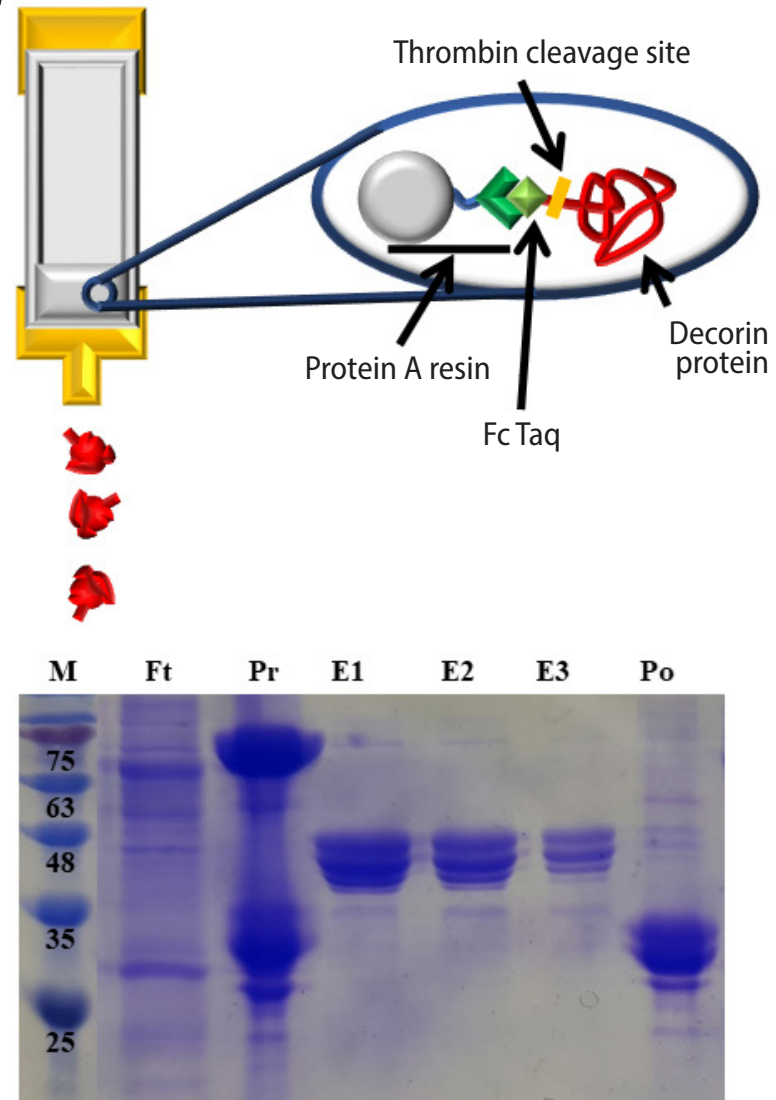

B

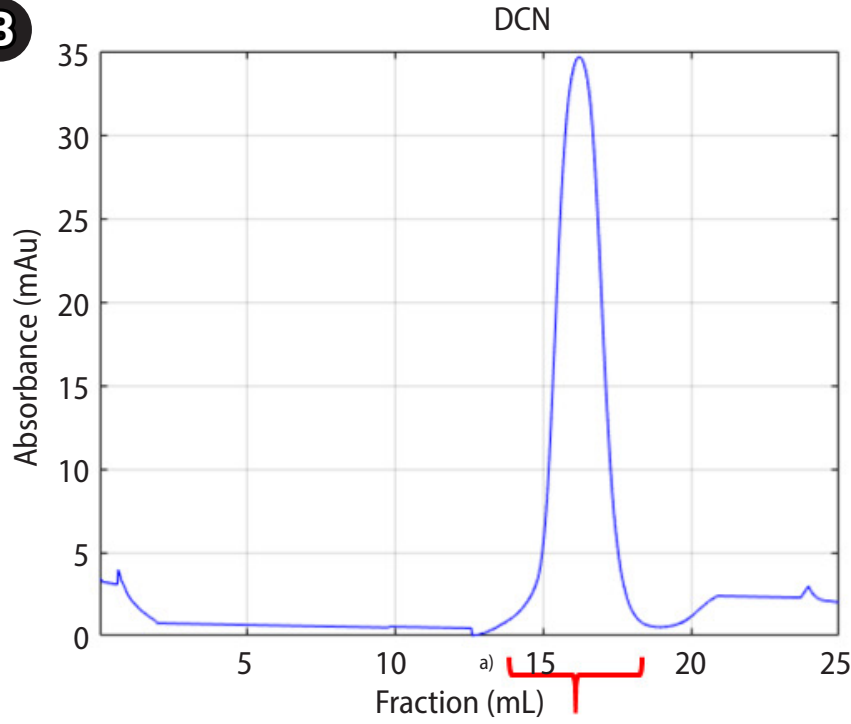

$\begin{array}{lllllllllllll}M & 11 & 12 & 13 & 14 & 15 & 16 & 17 & 18 & 19 & 20 & 21 & 22\end{array}$

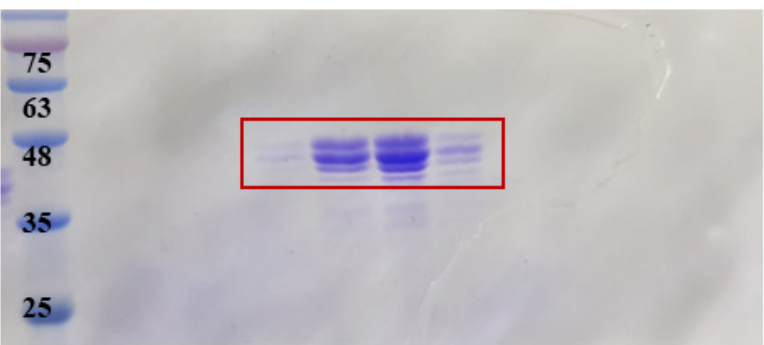

Figure 2. Production of the recombinant decorin (DCN) protein in insect cells. Recombinant human DCN was generated by a modified pAcGP67a vector. It was purified by affinity chromatography and size exclusion chromatography (SEC). (A) Scheme of affinity chromatography using protein A resin and sodium dodecyl sulfate-polyacrylamide gel electrophoresis (SDS-PAGE) gel showing the purity of DCN. Lane 1: marker (M); lane 2: flow-through (Ft); lane 3: pre-resin (Pr), which was DCN-Fc bound protein A resin before thrombin treatment; lanes 4-6: elutions (E1-E3) of DCN after thrombin treatment; lane 7: post-resin (Po), which was the remaining resin after elutions of DCN. (B) Elution profiles of SEC and SDS-PAGE gel showing the purity of DCN. For SDS-PAGE analysis, lane 1: marker (M); lane 2-12: fraction numbers of

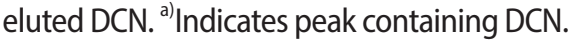

controlled ovarian stimulation protocols, the median level of F-DCN was slightly higher than that in the serum (S-DCN). However, F-DCN showed a weak negative correlation with S-DCN. They suggested that DCN actively taken into the follicular fluid from blood might play beneficial roles in follicle and oocyte maturation. Regardless of the fertilization method, fertilized eggs showed no significant differences between F-DCN and S-DCN. Interestingly, F-DCN of fertilized oocytes was significantly lower than that of unfertilized oocytes only in patients who underwent intracytoplasmic sperm injection. They also proved that the F-DCN level in intracytoplasmic sperm injection patients had potential to predict fertilization success based on a receiver operating characteristic (ROC) curve analysis. When they established a cut-off level of $34.5 \mathrm{ng} / \mathrm{mL}$ for F-DCN based on the ROC curve, they suggested that oocytes from follicles with F-DCN lower than the cut-off level tended to be better than those from oocytes with a high F-DCN [30].

\section{DCN in the uterus and trophoblast migration}

For successful blastocyst implantation, invasive trophoblast cells should mediate embryonic migration into the decidual matrix, forming abundant networks connecting embryonic tissue to maternal blood vessels. Signaling in pregnancy can induce the differentiation of endometrial stromal cells into decidual cells. Human decidual cells stimulated by steroid hormones can produce 2 SLRPs: DCN and biglycan. The maintenance of pregnancy is guided by the composition and organization of the endometrial ECM in the uterus. Certain pathological conditions that occur during pregnancy, including $P E$, have been linked to abnormal placental morphology and consequent fetal morbidity. In the uterus of a knockout mouse model, 


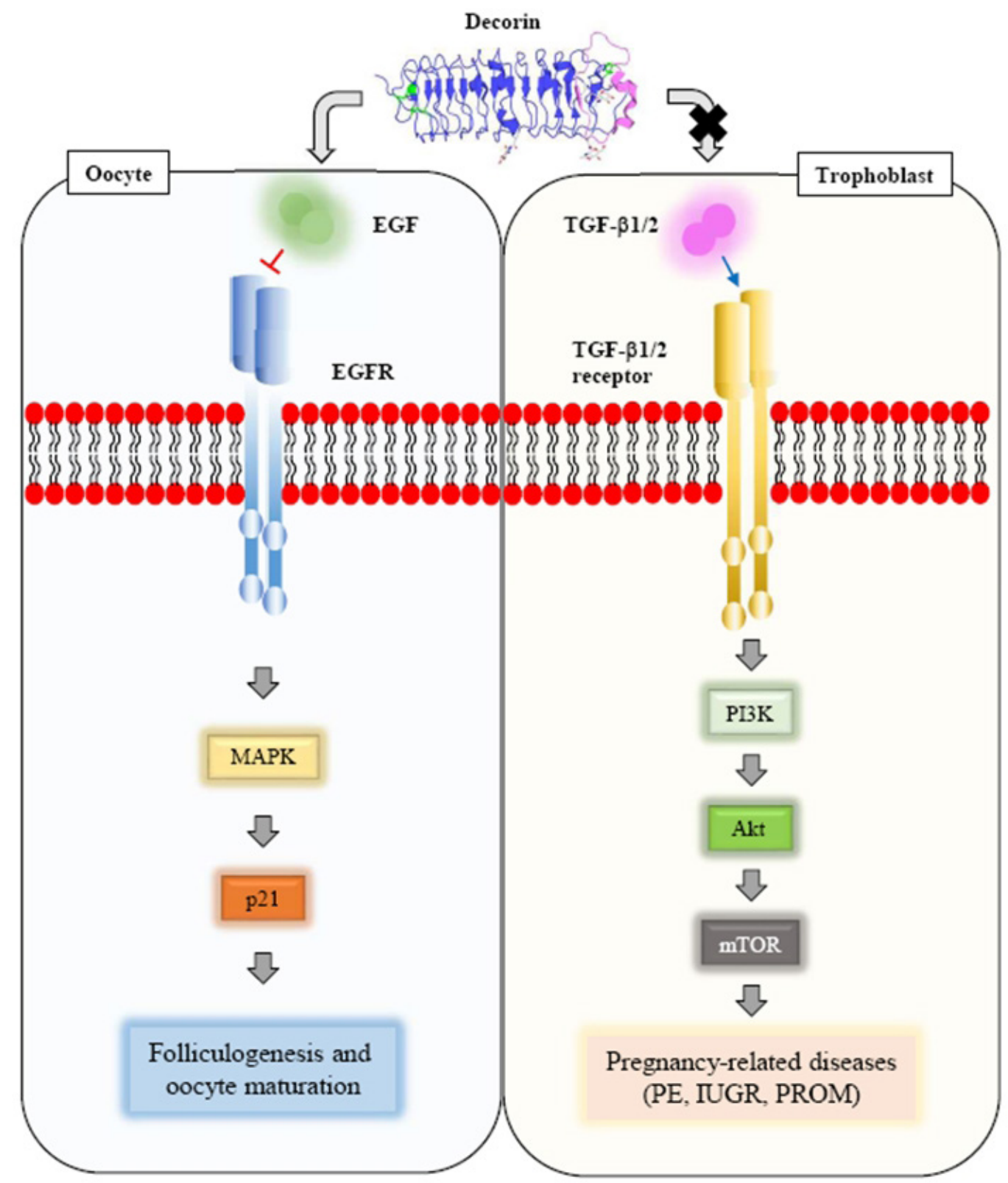

Figure 3. Schematic diagram showing how decorin acts as a multifunctional molecule during oocyte maturation and embryo implantation. EGF, growth factor receptor; EGFR, epidermal growth factor receptor; TGF, transforming growth factor; PE, preeclampsia; IUGR, intrauterine growth restriction; PROM, premature rupture of membranes.

DCN was found to be required for myometrium contraction in a DCN concentration-dependent manner, whereas biglycan exhibited partial compensation for DCN loss [31].

Transforming growth factor (TGF)- $\beta$ and DCN are produced in the human feto-maternal interface. They play decisive roles in the regulation of trophoblast invasion in the uterus. TGF- $\beta$ has DCN binding sites, and its activity is controlled by DCN. Lysiak et al. [32] reported that TGF- $\beta$ and DCN were co-localized in the ECM of first-trimester decidual tissues. They suggested that DCN might inhibit the activity of TGF- $\beta$ in the ECM of the placenta. In addition, it has been suggest- ed that migration, proliferation, and invasion of EVTs and choriocarcinoma cells are independently regulated by interactions between TGF- $\beta$ and DCN in decidual tissues [33]. At the human feto-maternal interface, the decidua forms a dense ECM structure that can regulate trophoblast invasion. Experimentally, silencing of KAI1 (a metastasis suppressor) by double-stranded RNA interference reduced expression of DCN, a decidual product implicated in limiting trophoblast invasion. It has been shown that KAl1 is expressed in decidual cells at the feto-maternal interface, where it might participate in the control of trophoblast invasion [34]. 
Several reports have shown that DCN in decidual tissue could regulate migration, proliferation, and invasion of EVTs of the human placenta in a TGF- $\beta$-independent manner [33-35]. These functions were differentially mediated by the binding of DCN to various tyrosine kinase receptors, including IGFR1, EGFR, and VEGFR2 [35]. It has been found that the overexpression of DCN in basal decidual cells is associated with a hypo-invasive phenotype and poor endovascular differentiation of trophoblast cells in PE [36]. Supplementation of DCN and knockdown of c-Met can reduce the proliferation and invasion in HTR-8 trophoblast cells. However, induction of autophagy and apoptosis by DCN were not synergistically enhanced by c-Met knockdown. It was found that DCN could promote autophagy and apoptosis mainly through downregulating c-Met/Akt/mTOR activity in human trophoblast cells [10].

Halari et al. [37] found that DCN production increased during the decidualization of human endometrial stromal cells in vitro and early gestation in decidual samples tested ex vivo. Endometrial stromal cell maturation and differentiation into decidual cells are crucial for a normal pregnancy. In their study, depleting DCN in human endometrial stromal cells treated with decidualization stimulation failed to induce morphologically and functionally appropriate maturation and differentiation of decidual cells [37]. Recently, it has been suggested that 2 SLRPs, DCN and biglycan, play important roles in the structural and functional integrity of the placenta and fetal membrane and that their alterations may lead to several pregnancy-related diseases such as repeated implantation failure, PE, IUGR, and premature rupture of membranes [4].

\section{Conclusion}

DCN is a multifunctional molecule. Its functions are mediated by a variety of binding events, including receptor-mediated and glycosaminoglycan-mediated binding. At the human feto-maternal interface, TGF- $\beta$ and DCN play crucial roles in the regulation of trophoblast invasion in the uterus. TGF- $\beta$ has DCN binding sites. Its activity is controlled by DCN. During pregnancy, orchestrated hormonal control of successful pregnancy should regulate the concentration and activity of specific proteins such as DCN participating in the ECM remodeling of trophoblastic and uterine cells. This review confirms that proteoglycan DCN is an important and multifunctional molecule in the physiological regulation of oocyte maturation and trophoblast migration. The findings discussed herein suggest that recombinant DCN proteins might be useful for substantiating these diverse functions in both animal and in vitro models of oogenesis and implantation (Figure 3 ).

\section{Conflict of interest}

No potential conflict of interest relevant to this article was reported.

\section{Acknowledgments}

We thank Ho min Kim (from the IBS and KAIST) for his help with protein expression.

\section{ORCID}

Beom Seok Park ～https://orcid.org/0000-0003-1208-1517

Jaewang Lee https://orcid.org/0000-0001-6801-7149

Jin Hyun Jun

https://orcid.org/0000-0001-9898-4471

\section{Author contributions}

Conceptualization: all authors. Data curation: all authors. Formal analysis: all authors. Funding acquisition: BSP. Methodology: all authors. Project administration: all authors. Visualization: all authors. Writing-original draft: all authors. Writing-review \& editing: all authors.

\section{References}

1. lozzo RV, Schaefer L. Proteoglycan form and function: a comprehensive nomenclature of proteoglycans. Matrix Biol 2015;42:1155.

2. Scott PG, McEwan PA, Dodd CM, Bergmann EM, Bishop PN, Bella J. Crystal structure of the dimeric protein core of decorin, the archetypal small leucine-rich repeat proteoglycan. Proc Natl Acad Sci U S A 2004;101:15633-8.

3. Park BS, Lee JO. Recognition of lipopolysaccharide pattern by TLR4 complexes. Exp Mol Med 2013;45:e66.

4. Halari CD, Zheng M, Lala PK. Roles of two small leucine-rich proteoglycans decorin and biglycan in pregnancy and pregnancy-associated diseases. Int J Mol Sci 2021;22:10584.

5. Kajava AV. Structural diversity of leucine-rich repeat proteins. J Mol Biol 1998;277:519-27.

6. Jin MS, Lee JO. Application of hybrid LRR technique to protein crystallization. BMB Rep 2008;41:353-7.

7. Park BS, Won SY, Lee DS, Kim HM. Human leucine-rich-alpha-2-glycoprotein1: purification, crystallization, and X-ray crystallographic analysis. Biodesign 2020;8:60-3.

8. Franczyk M, Wawrzykowski J, Kankofer M. Preliminary results of the placental decorin profile in bovine pregnancy and parturition. 
Glycoconj J 2018;35:461-5.

9. Danielson KG, Baribault H, Holmes DF, Graham H, Kadler KE, lozzo RV. Targeted disruption of decorin leads to abnormal collagen fibril morphology and skin fragility. J Cell Biol 1997;136:729-43.

10. Wang Y, Zhang H, Zhang Y, Li X, Hu X, Wang X. Decorin promotes apoptosis and autophagy via suppressing c-Met in HTR-8 trophoblasts. Reproduction 2020;159:669-77.

11. Jamioł M, Wawrzykowski J, Kankofer M. The influence of progesterone and prostaglandin F2a on decorin and the adhesion of caruncular epithelial cells of bovine placenta at early-mid pregnancy-Part II. Reprod Domest Anim 2021;56:1040-9.

12. Ricciardelli C, Rodgers RJ. Extracellular matrix of ovarian tumors. Semin Reprod Med 2006;24:270-82.

13. Grisaru D, Hauspy J, Prasad M, Albert M, Murphy KJ, Covens A, et al. Microarray expression identification of differentially expressed genes in serous epithelial ovarian cancer compared with bulk normal ovarian tissue and ovarian surface scrapings. Oncol Rep 2007; 18:1347-56.

14. Santra M, Reed CC, lozzo RV. Decorin binds to a narrow region of the epidermal growth factor (EGF) receptor, partially overlapping but distinct from the EGF-binding epitope. J Biol Chem 2002; 277:35671-81.

15. Park JY, Su YQ, Ariga M, Law E, Jin SL, Conti M. EGF-like growth factors as mediators of $\mathrm{LH}$ action in the ovulatory follicle. Science 2004;303:682-4.

16. Ben-Ami I, Freimann S, Armon L, Dantes A, Strassburger D, Friedler $S$, et al. PGE2 up-regulates EGF-like growth factor biosynthesis in human granulosa cells: new insights into the coordination between PGE2 and LH in ovulation. Mol Hum Reprod 2006;12:5939.

17. lozzo RV, Schaefer L. Proteoglycans in health and disease: novel regulatory signaling mechanisms evoked by the small leucine-rich proteoglycans. FEBS J 2010;277:3864-75.

18. Csordás G, Santra M, Reed CC, Eichstetter I, McQuillan DJ, Gross D, et al. Sustained down-regulation of the epidermal growth factor receptor by decorin: a mechanism for controlling tumor growth in vivo. J Biol Chem 2000;275:32879-87.

19. Zhu JX, Goldoni S, Bix G, Owens RT, McQuillan DJ, Reed CC, et al. Decorin evokes protracted internalization and degradation of the epidermal growth factor receptor via caveolar endocytosis. J Biol Chem 2005;280:32468-79.

20. Adam M, Schwarzer JU, Köhn FM, Strauss L, Poutanen M, Mayerhofer A. Mast cell tryptase stimulates production of decorin by human testicular peritubular cells: possible role of decorin in male infertility by interfering with growth factor signaling. Hum Reprod 2011;26:2613-25.

21. lozzo RV, Moscatello DK, McQuillan DJ, Eichstetter I. Decorin is a biological ligand for the epidermal growth factor receptor. J Biol Chem 1999;274:4489-92.

22. Ben-Ami I, Freimann S, Armon L, Dantes A, Ron-El R, Amsterdam A. Novel function of ovarian growth factors: combined studies by DNA microarray, biochemical and physiological approaches. Mol Hum Reprod 2006;12:413-9.

23. Yu Y, Yan J, Li M, Yan L, Zhao Y, Lian Y, et al. Effects of combined epidermal growth factor, brain-derived neurotrophic factor and insulin-like growth factor-1 on human oocyte maturation and early fertilized and cloned embryo development. Hum Reprod 2012; 27:2146-59.

24. Adam M, Saller S, Ströbl S, Hennebold JD, Dissen GA, Ojeda SR, et al. Decorin is a part of the ovarian extracellular matrix in primates and may act as a signaling molecule. Hum Reprod 2012;27:324958.

25. Peng JY, Gao KX, Xin HY, Han P, Zhu GQ, Cao BY. Molecular cloning, expression analysis, and function of decorin in goat ovarian granulosa cells. Domest Anim Endocrinol 2016;57:108-16.

26. Kedem A, Ulanenko-Shenkar K, Yung Y, Yerushalmi GM, Maman E, Hourvitz A. Elucidating Decorin's role in the preovulatory follicle. J Ovarian Res 2020;13:15.

27. Richards H, Murley C, Poat JA, Munday KA. Proceedings: studies on levels of angiotensin II in the circulation of the rat. J Endocrinol 1976;68:11-2.

28. Maman E, Yung Y, Kedem A, Yerushalmi GM, Konopnicki S, Cohen $B$, et al. High expression of luteinizing hormone receptors messenger RNA by human cumulus granulosa cells is in correlation with decreased fertilization. Fertil Steril 2012;97:592-8.

29. Yung Y, Aviel-Ronen S, Maman E, Rubinstein N, Avivi C, Orvieto R, et al. Localization of luteinizing hormone receptor protein in the human ovary. Mol Hum Reprod 2014;20:844-9.

30. Sawada Y, Sato T, Saito C, Ozawa F, Ozaki Y, Sugiura-Ogasawara M. Clinical utility of decorin in follicular fluid as a biomarker of oocyte potential. Reprod Biol 2018;18:33-9.

31. Wu Z, Aron AW, Macksoud EE, lozzo RV, Hai CM, Lechner BE. Uterine dysfunction in biglycan and decorin deficient mice leads to dystocia during parturition. PLoS One 2012;7:e29627.

32. Lysiak JJ, Hunt J, Pringle GA, Lala PK. Localization of transforming growth factor beta and its natural inhibitor decorin in the human placenta and decidua throughout gestation. Placenta 1995;16: 221-31.

33. Xu G, Guimond MJ, Chakraborty C, Lala PK. Control of proliferation, migration, and invasiveness of human extravillous trophoblast by decorin, a decidual product. Biol Reprod 2002;67:681-9.

34. Gellersen B, Briese J, Oberndorfer M, Redlin K, Samalecos A, Richter DU, et al. Expression of the metastasis suppressor KAl1 in decidual cells at the human maternal-fetal interface: regulation and 
functional implications. Am J Pathol 2007;170:126-39.

35. Nandi P, Siddiqui MF, Lala PK. Restraint of trophoblast invasion of the uterus by decorin: role in pre-eclampsia. Am J Reprod Immunol 2016;75:351-60.

36. Siddiqui MF, Nandi P, Girish GV, Nygard K, Eastabrook G, de Vrijer B, et al. Decorin over-expression by decidual cells in preeclampsia: a potential blood biomarker. Am J Obstet Gynecol 2016;215:361 . e1-361.e15.

37. Halari CD, Nandi P, Jeyarajah MJ, Renaud SJ, Lala PK. Decorin production by the human decidua: role in decidual cell maturation. Mol Hum Reprod 2020;26:784-96. 